\title{
Entre parents et enfants : liens et relations à l'épreuve du cheminement vers la vie adulte
}

\author{
\begin{tabular}{c|l} 
& \\
Claire Bidart & Chargée de recherche au CNRS, LEST (UMR 6123).
\end{tabular} \\ Anne Pellissier Maître de conférences à l'université de Caen, \\ CERSE (EA 965).
}

Listan e processus de cheminement vers l'âge adulte -évolue et transforme les rapports entre les jeunes et leurs parents. Leurs relations deviennent-elles pour autant individualisées et électives ou persistet-il quelque chose de ce lien "structurel ", lié à des places symboliques résistant au temps? Comment se nouent la relation affinitaire entre deux adultes et le lien inamovible entre parents et enfants ? À partir d'une enquête longitudinale qui suit un panel de jeunes sur plusieurs années, cet article montre que les « liens » de parenté conservent, en particulier dans des contextes familiaux douloureux et conflictuels, un caractère inamovible. Les "relations" avec les parents sont, elles, plus librement et diversement mises en œuvre; le passage à la vie adulte les voit évoluer vers une plus grande personnalisation. Ces évolutions montrent pourtant certaines ambivalences. Pour ces jeunes, le fait d'avoir un enfant se révèle très fortement imbriqué dans leurs rapports avec leurs parents, ce qui conduit à privilégier la « double dimension intergénérationnelle ».

Les conditions historiques dans lesquelles se construisent les générations imprègnent leur "destin » et influent sur les modalités du passage d'une génération à la suivante (Chauvel, 1998). Ces conditions économiques, politiques, démographiques, etc., transforment les rapports entre les générations, notamment les relations entre les parents et les enfants (Chamboredon, 1985). De ce point de vue, les deux sens du mot "génération » sont impliqués au moment du passage à l'âge adulte dans la mesure où la jeunesse constitue non seulement la charnière entre les générations historiques mais aussi le pivot du processus de transmission. Elle se trouve donc au cœur de ces mutations. On a pu ainsi observer l'émergence d'un "nouvel âge de la vie ", résultant de l'allongement de l'intervalle entre l'état de dépendance de l'enfant envers ses parents et la conquête définitive de son autonomie (Galland, 1990). Le constat s'est ensuite précisé : la transition de la jeunesse à l'âge adulte s'est non seulement allongée mais elle s'est également complexifiée, dissociée, fragmentée. Elle s'est diversifiée aussi, comme l'attestent les différences selon les pays et les cultures (Galland, 2001; Cicchelli, 2001 a ; Van de Velde, 2004 ; Bidart, 2006). On observe également des disparités au sein d'un même pays, entre les sexes et entre les classes sociales en particulier. On ne peut donc parler d'une seule jeunesse, d'un seul modèle de transition intergénérationnelle.

\section{De nouveaux modes de cheminement vers l'âge adulte}

Cette diversification et cette fragmentation ont pu faire penser à une individualisation, voire à une singularisation des parcours : à chacun son destin, son itinéraire, sa jeunesse... Pourtant, les différenciations sociales et l'impact de la transformation des rapports entre générations rappellent que ces transitions, même plus difficiles à synthétiser, sont loin d'être dégagées des déterminations historiques et des rapports sociaux (Mauger, 1994 ; Battagliola, 1997 ; Bidart et Lavenu, 2006). Cette diversité, cette complexité croissante ont également modifié le regard que portent les sciences sociales sur ces transitions. Plus que d'un « allongement » de la jeunesse, on parle maintenant de nouveaux modes de cheminement vers l'âge adulte, signalant davantage des formes nouvelles de socialisation que des « moratoires » (Blöss, 1997). Ainsi, les rapports entre les « jeunes adultes » et leurs parents doivent-ils être analysés en tenant compte de la plus grande diversité de comportements et de relations entre les parents et les enfants, et pas seulement comme un report du départ du nid familial.

Il convient, dès lors, d'étudier le cheminement vers l'âge adulte moins comme une période d'accès à l'indépendance que comme une période de renégociation des places entre la génération des parents et celle des enfants. On parlera d'une relation "dialectique », d'une " transaction » plus que d'une 
«transmission » intergénérationnelle (Dubar, 1995). En effet, les jeunes sont de plus en plus susceptibles de construire une autonomisation " négociée » dans le cadre de leur famille d'origine (Cicchelli, 2001 b ; Ramos, 2002). La cohabitation entre les parents et un " jeune adulte » est plus longue, mais incorpore également diverses étapes relativement désynchronisées: la fin des études, l'entrée dans le monde du travail (Rose, 1998) (parfois suivie d'une sortie par le chômage), la formation d'un couple (Le Gall et Pellissier, 2001) et son éventuelle rupture, I'indépendance financière (et sa perte éventuelle). Les retours chez les parents, sans pour autant signifier un retour en arrière, sont plus fréquents (Pellissier, 2002). Dans cette optique, le "pacte» qui lie le jeune et ses parents (Cicchelli, 2001 a), tout au long de cette période où l'indépendance des jeunes n'est pas assurée alors que leur autonomie se construit (Singly, 2000), prend des formes nouvelles.

Ces réaménagements sont favorisés par la conception moderne des liens, qui fonde de plus en plus leur légitimité sur le libre arbitre des individus et sur la qualité affective de la relation. Devenues "électives », les relations familiales seraient donc de plus en plus " choisies » par des individus déchargés de la pesanteur des conventions et des prescriptions attachées aux liens de parenté. Ainsi, selon François de Singly (2003), si la modernité n'a pas libéré les individus des contraintes familiales, elle leur permet cependant de décider "librement » de celles qu'ils acceptent et de celles qu'ils rejettent. Cependant, même en tenant compte de la personnalisation et de la psychologisation des relations entre parents et enfants, pour d'autres auteurs le "pacte de filiation » reste un lien inconditionnel, non négociable (Théry, 1998).

\section{Devenir adulte... avec ses parents}

Cet article propose de revenir sur ce débat en distinguant deux modalités du rapport intergénérationnel : le lien, qui désigne la place et le rôle symboliques, anthropologiques et structurels des parents et de leurs enfants, et la relation, c'est-àdire la manière conjoncturelle de vivre la parenté et d'interagir avec ses parents et ses enfants. La famille implique en effet à la fois un lien et une relation, et le rapport entre parents et enfants se joue sur ces deux tableaux : celui du lien, qui reste quoi qu'il arrive une donnée inaltérable et inconditionnelle ; et celui de la relation, d'intensité variable, qui peut évoluer, qui est sensible aux événements et à I'histoire familiale. La réflexion présentée ici est construite à partir d'une enquête longitudinale auprès d'un panel d'une soixantaine de jeunes réinterrogés tous les trois ans depuis 1995, soit depuis bientôt douze ans (encadré). Sont principalement traités les modes de socialisation et les évolutions des réseaux relationnels au fil du cheminement de ces jeunes vers I'âge adulte.

Comment devient-on adulte ? Envisager le passage à l'âge adulte comme un processus, c'est retrouver la dimension intergénérationnelle qui l'accompagne. Quel rôle jouent les parents dans ces transitions biographiques? Comment évoluent les rapports avec eux? L'impact de ce rapport intergénérationnel dans le cheminement vers l'âge adulte s'avère crucial. Ce dernier revêt en effet un caractère très fortement interactif : on est adulte dans certains domaines, avec certaines personnes et pas avec d'autres, on devient adulte sous le regard de certains "autruis significatifs", et principalement de ses parents. Cette transition engage une transformation du lien entre les générations, mais aussi des relations entre parents et enfants. On peut s'interroger alors ici sur le caractère "négociable » du lien entre générations, ainsi que sur la possibilité de "faire le tri » par rapport à l'héritage familial, en signalant que ces ouvertures ne sont pas distribuées également dans la société. Si l'ensemble de la famille étendue peut être concernée, il sera principalement traité ici des liens les plus pertinents pour la transition vers l'âge adulte, à savoir les liens entre parents et enfants.

\section{Les liens de famille : l'incontournable parenté}

Les liens de famille, mais aussi les relations avec les parents, ont un impact certain sur la socialisation d'un jeune devenant un adulte. Les rapports avec les parents agissent sur les orientations, sur les transitions, sur les parcours, et inversement les événements de la vie, les expériences, les cheminements agissent sur les relations avec les parents. On voit ainsi les liens avec les parents dans leur dimension symbolique, mais également les relations entretenues et transformées avec eux, se manifester et prendre leur part dans les processus de passage à la vie adulte. Dans les rapports avec la famille, certaines obligations restent, semble-t-il, incontournables. Les parents ne sont pas les seuls concernés. Souvent, en effet, les membres du réseau de parenté se fréquentent non parce qu'ils s'aiment (même s'ils peuvent s'aimer), mais parce qu'ils appartiennent à la même famille. Les jeunes témoignent de cette part de contrainte, comme le fait Mélanie : "Tout ce qui est repas de famille, je déteste. Parce que c'est interminable et beaucoup de gens détestent, mais aussi ça force des gens qui n'ont pas spécialement envie de se retrouver, genre la belle-sœur qui peut pas saquer l'autre. C'est un peu les contacts: "bon, on s'aime pas mais comme on fait partie de la même famille, on se voit". C'est partout pareil mais c'est dommage. 


\section{Méthodologie de l'enquête}

Il s'agit d'une enquête qualitative en cours auprès d'un panel de jeunes vivant à l'origine dans l'agglomération de Caen en Normandie. Ont été interrogés des jeunes au seuil d'une étape importante, à savoir le baccalauréat ou la fin d'un stage $d^{\prime}$ insertion. Des entretiens approfondis ont été réalisés auprès de ces jeunes.

La population de l'enquête a été sélectionnée sur deux critères : la filière scolaire suivie et le sexe. En 1995, 87 jeunes ont été interviewés une première fois, dont un tiers en classe terminale de la section économique et sociale (bac ES), un tiers en classe de LEP (bac professionnel), et un tiers en stage d'insertion. Filles et garçons ont été répartis par moitié dans chacun des trois groupes. Trois ans après, en 1998, ils ont été à nouveau contactés et 73 d'entre eux ont été réinterrogés. Encore trois ans après, en 2001, 66 de ces jeunes ont à nouveau participé à l'enquête, puis 60 en 2004. Une interrogation plus succincte et ciblée est réalisée en 2007. Les jeunes qui ont abandonné l'enquête sont surtout des garçons peu diplômés. En première vague d'enquête, ces jeunes étaient âgés de 17 ans à 23 ans. Trois ans après, certains poursuivent des études, d'autres travaillent, sont au chômage ou dans d'autres situations encore. Encore trois ans après, ils avancent toujours vers la vie adulte, certains vivent encore chez leurs parents, d'autres seuls ou en couple, certains ont des enfants... À chaque fois, ils sont réinterrogés là où ils vivent. La méthode d'enquête combine des questionnaires retraçant leurs trajectoires mois par mois sur des calendriers, en cumulant les informations dans les domaines professionnel, familial, résidentiel, amoureux, associatif et en repérant l'ensemble des événements importants survenus dans ces trois ans. Les réseaux relationnels sont construits à partir de séries de questions posées à propos des divers contextes de vie abordés (études, travail, loisirs, famille, voisinage, etc.). Les caractéristiques sociographiques de ces divers partenaires sont recueillies par la suite, ainsi que des descriptions des relations.

Suivent des entretiens qualitatifs approfondis dans lesquels sont longuement discutés les événements et mutations tant relationnels que biographiques. On a donc poursuivi l'étude des processus d'insertion selon les axes problématiques qui ont défini ce projet de recherche, dès ses origines: l'étude des interactions entre les diverses sphères de la vie (travail, famille, couple, résidence, mobilités, loisirs...) dans la construction des trajectoires des jeunes; l'analyse de leurs réseaux relationnels en tant que facteurs de socialisation; la prise en compte de la dimension diachronique de leurs avancées vers la vie d'adulte.

Cette enquête est réalisée par Claire Bidart, Alain Degenne, Daniel Lavenu, Didier Le Gall, Lise Mounier et Anne Pellissier. Elle s'inscrit dans une coopération entre le Laboratoire d'économie et de sociologie du travail, le Centre Maurice Halbwachs et le Centre d'études et de recherche en sciences de l'éducation de I'Université de Caen-BasseNormandie, des laboratoires qui associent des universités et le CNRS. Elle a été financée entre autres par la Délégation interministérielle à l'insertion des jeunes (ministère de l'Emploi et de la Solidarité), la direction régionale des Affaires sanitaires et sociales de Basse-Normandie, la direction départementale des Affaires sanitaires et sociales du Calvados, France Télécom R\&D et la Caisse nationale des allocations familiales.

Pour en savoir plus :

http://halshs.archives ouvertes.fr/halshs-00118258
Moi, j'essaie d'avoir des liens, ne serait-ce que parce que, par rapport à ça, j'ai pas mal de principes, je dirais que, par rapport au fait que ça soit ma famille, il faut que je la respecte quoi qu'il arrive... ». Le caractère électif des relations avec la parenté montre ici sa limite, celle des "principes» qu'évoque Mélanie et que l'on peut référer au « lien de famille».

Émeline distingue également ces deux modalités du rapport à la famille : même si les membres en sont diversement appréciés, on doit néanmoins tenir compte de certaines positions : "Ma grandmère Marguerite, pour le coup, c'est vraiment conventionnel. Ma grand-mère, je la vois très peu. Je ne suis pas sa petite-fille préférée, c'est clair. Elle adore ma petite sœur. Enfin, je ne lui en veux pas du tout. Mais elle compte pour moi parce que je sais que c'est le noyau familial de ma famille et, maintenant, elle est très âgée. Le jour où elle disparaît, il va y avoir une remise en cause terrible, c'est clair».

Les rapports collatéraux ne font pas exception et Denis, dans la relation avec son frère, distingue clairement le rapport familial du rapport affinitaire: - «Tu imagines ne plus avoir de relations avec ton frère?" - "Non, pas aucune, mais ça ne sera que familial. On n'a pas d'affinités. On est très différents l'un de l'autre ". Denis montre bien en quoi le lien familial résiste au principe d'élection, aux sentiments, aux choix. Institutionnalisés, inamovibles, les liens familiaux ne sont pas tout à fait des relations comme les autres. Persistant au-delà des affinités et de leurs aléas, ces conventions donnent une certaine " coloration » aux interactions qui $s^{\prime} y$ déroulent. Si Viviane apprécie les réunions familiales, elle témoigne en même temps de l'impossibilité d'y exprimer son identité personnelle : "J'adore les repas de famille, j'adore voir ma famille. J'ai besoin de mes amis pour délirer mais j'adore ma famille. J'aime bien être avec eux parce qu'ils m'ont vue grandir, je les ai vus grandir. Parce qu'on est bien ensemble, je pense que c'est ça. Mais ce qui me déplaît, c'est vrai que je ne peux pas vraiment être moi avec ma famille, c'est vrai qu'il y a une barrière, on est quand même respectueux, c'est quand même sa famille, il y a un respect à avoir ". Le lien, dans ces exemples, existe donc bien au-delà de l'expression individuelle et au-delà des fluctuations relationnelles. On le perçoit plus particulièrement à l'occasion d'une épreuve ou d'un éloignement. Ainsi, Nicolas, à qui I'on demande s'il se sent plutôt «famille » ou pas, répond-il : "Oui. Je I'ai découvert récemment, à cause de l'éloignement de ma famille, tout simplement, de ma famille proche. Je m'aperçois que je tiens vraiment à eux ». 
La " bonne distance " de la décohabitation

Étape du cheminement vers l'âge adulte, la décohabitation n'annihile pas le caractère en partie contraint, inaliénable du lien parental. Certes, bien des jeunes témoignent par exemple de l'apaisement que permet le fait de ne plus vivre sous le même toit. C'est le cas pour Gilles : «Ça a peutêtre été dur sur le coup pour mon père mais, le fait que je prenne un appart' a peut-être solutionné le problème relationnel qui pouvait exister entre nous. Ça pouvait être une solution trop facile, c'était peut-être un moyen d'éviter le dialogue et, à la limite, les clashs possibles entre nous. Mais, depuis que j'ai mon appart', ça fait deux ans, cela va beaucoup mieux. Je crois qu'on a mis tous les deux de l'eau dans notre vin et puis voilà ». La décohabitation est une occasion de mettre sur la table bien des choses, ou encore de les mettre à distance. II reste que cette décision se prend rarement sans les parents. Par ailleurs, le maintien de plus en plus fréquent $d^{\prime}$ 'une dépendance financière après le départ implique certes quelques contraintes, mais celles-ci n'annulent pas le processus d'autonomisation (Singly, 2000).

Pour autant, la manière dont les visites s'organisent après le départ du domicile parental témoigne de la résistance du lien intergénérationnel à cette transformation du mode d'interaction. Certes, la décohabitation rend les relations avec les parents plus "électives »: on ne leur rend visite que lorsqu'on le décide, et ces rencontres sont d'autant plus appréciées qu'elles répondent désormais à un choix et non à une contrainte. Cependant, des différences apparaissent au regard de l'origine sociale. Les visites aux parents se prévoient sur un rythme régulier dans les classes populaires : «On s'est donné ça, avec Marjorie, de voir nos parents au moins une fois par semaine, même plus, mais minimum une fois par semaine", explique Yves. Les jeunes de milieu aisé refusent davantage de formaliser le rythme des visites. Antoine, qui habite encore chez ses parents, imagine ainsi la relation qu'il aura avec eux quand il aura décohabité : "Le rituel du repas dominical, non merci! Au moins, j'irai passer un week-end chez eux parce que je sais que la porte sera toujours ouverte, et je serai content d'être avec eux. (...) J'adore être avec mes parents quand j'ai envie d'être avec eux!». Le mode "électif » semble ainsi se revendiquer plus clairement dans les catégories sociales supérieures, pour la parenté comme pour l'amitié (Bidart, 1997).

Cependant, quelle que soit l'origine sociale, les visites aux parents, même relativement formalisées, ne sont pas franchement décrites par les enquêtés en terme d'obligation tant l'expression de la contrainte paraîtrait altérer le lien affectif entre parents et enfants mais aussi leur liberté de jeunes adultes. Ainsi, de la même façon que les formes de la sociabilité doivent pour Georg Simmel (1981) faire " oublier » les contraintes sociales, en matière familiale la contrainte doit se faire invisible ou être rapportée à un choix du sujet, comme l'exprime Émeline lorsqu'on lui demande si elle se sent des obligations à l'égard de ses parents : "Obligée, je me sens des obligations morales, mais qui ne me pèsent pas. Je le fais et c'est encore de bon cœur ». En effet, même en douceur, la prescription apparaît. Celle-ci n'est pas toujours explicitement énoncée par les parents mais plutôt suggérée; les enfants, tout en affirmant visiter leurs parents quand ils en ont envie, se révèlent souvent sensibles à leurs « suggestions ». Thibaut résume bien toute I'ambivalence de la situation :

"Ce n'est pas une obligation, mais je le fais parce que je me mets aussi à leur place, surtout ma mère, je vais les voir au moins une fois par semaine. Je ne peux pas dire que c'est une obligation, mais en même temps je me sens un peu obligé. Ça ne me déplaît pas $d^{\prime} y$ aller, mais il faut que je pense à y aller au moins une fois. Si je $n^{\prime} y$ vais pas une fois, ma mère ne va pas être bien et c'est là que ça va peut-être recréer des tensions. Ce n'est pas méchant de sa part, mais elle a besoin de nous voir. Je pense que c'est tout le monde pareil. On n'a pas le choix. C'est quand même nos parents. Je pense qu'on sera content que nos enfants viennent nous voir aussi. Je pense que c'est normal. Tout ne peut pas être parfait non plus ».

Si la décohabitation rend les relations avec les parents plus électives, elle ne supprime pas toute part de contrainte. Cependant, en raison de I'autonomisation du jeune adulte, celle-ci ne peut être reconnue en tant que telle, ni par les parents ni par les enfants. De manière plus générale, cette situation paradoxale renvoie à l'ambivalence du rapport à la famille en régime «individualiste ». Si, comme le montre François de Singly (2003), les relations familiales tendent - à l'exception de celles avec les tout petits enfants - à devenir de plus en plus "électives » et " contractuelles », cette évolution masque mais n'annule pas la persistance d'une part de normativité et de contrainte dans les liens et les relations entre parents et enfants.

Le poids du lien dans les rapports douloureux Il a été surprenant de découvrir, dans une enquête dont ce n'était pas la destination première, I'ampleur et l'impact des relations douloureuses entre parents et enfants. Les problèmes de famille sont en effet au cœur de bien des rapports intergénérationnels, grevant à la fois les relations et les parcours. Ces difficultés prennent divers aspects : parfois conflit global divisant l'ensemble de la famille étendue depuis des générations et porté avec beaucoup de honte par les jeunes qui en héritent (Le Gall, 1998) ; parfois recompositions 
familiales les laissant en conflit avec un de leurs parents ; parfois « secrets de famille » renvoyant à I'inceste, à la naissance illégitime, ou encore désaccord profond n'engageant " que » le jeune et ses parents, etc. Les cas de figure sont divers mais leur point commun se trouve dans leur impact sur les relations intergénérationnelles. On parle ici de relations « douloureuses » dans la mesure où, dans certains cas, ces difficultés vont avec des relations de désamour entre parents et enfants, mais dans $d^{\prime}$ autres cas se conjuguent avec des relations $d^{\prime}$ 'amour entre eux. La force et la persistance du lien parental se révèlent d'une manière particulièrement criante dans ces rapports douloureux. Dans un régime d'élection, ces relations devraient " logiquement » aboutir à leur rupture. Pourtant, les exemples sont foison de relations très problématiques maintenues, ou à l'inverse de relations rompues mais regrettées: le lien dans ces cas-là apparaît comme le nœud du rapport intergénérationnel, et parfois comme son calvaire.

Plutôt qu'une étape de la maturation du jeune, la décohabitation trouve alors souvent son origine dans le caractère insupportable des relations familiales. Ainsi, Marine ne veut retourner chez sa mère à aucun prix: "Ma mère, déjà, elle n’a jamais eu d'amour maternel envers moi. Moi je l'aimais et pas elle. (...). Maintenant, c'est fini. Après ce qu'elle a fait, c'est clair. Je n'ai plus de mère. Didier viendrait me dire que ma mère est mourante, je n'irais pas la voir. (...). Quand tu es dans ta famille, c'est terrible! Pour rien au monde... Je viendrais à me séparer [de son mari], je préfèrerais aller dans un foyer que d'aller chez ma mère!".

À l'inverse, dans certains cas les relations problématiques sont justement ce qui empêche la décohabitation. Ainsi, Diane se trouve perpétuellement tiraillée entre l'impossibilité d'abandonner sa mère alcoolique après le suicide de son père, et son désir de partir pour échapper à une situation familiale insoutenable. En seconde vague de l'enquête, elle explique : "J'aimerais bien rester à C. parce qu'il y a ma mère, et ma mère, j'aurais trop peur qu'elle... de la laisser toute seule en fait. Parce que je veux partir de chez moi, mais avec tout ce qu'elle a fait et tout ce que... tout ce que j'ai vu, j'aurais trop peur qu'elle reste toute seule (...). Mais j'aimerais bien partir quand même, peut-être juste un an... ». Plus tard, Diane part vivre avec Marc, mais retourne rapidement chez sa mère. Quand on la retrouve quelques années après, en quatrième vague d'enquête, elle vit à nouveau avec Marc et attend un enfant. Elle n'est pas pour autant détachée de sa mère et l'éloignement, bien que nécessaire, reste difficile : "J'avais besoin de faire ma vie (...). Mais j'ai peur qu'il arrive quelque chose à ma mère, et que je ne sois pas là. Le fait que, pour mon père, j'étais trop jeune, je n'ai rien compris à ce qui se passait et on n'a rien vu venir, eh bien je me dis qu'avec ma mère, il ne faut pas que ça arrive. Donc ça arrive tellement vite que je veux être là. Je voudrais être là tout le temps! ».

\section{La difficulté à " couper les ponts "}

La décohabitation ne signifie pas alors qu'il ait été mis fin à la relation, ni que celle-ci se soit stabilisée sur un certain mode, mais inaugure plutôt une série de hauts et de bas, de périodes où l'on voit ses parents et de périodes où l'on n'a plus de contacts, de disputes suivies de réconciliations. Marine qui, en deuxième vague d'enquête, déclarait ne plus jamais vouloir voir sa mère, explique lors de la troisième vague : "Avec ma mère, cela a toujours été comme ça. Il y a des hauts et des bas. D'un seul coup ça va, d'un seul coup, ça ne va plus... (...). Maintenant, on mange ensemble. Elle vient manger chez moi ».

D’une manière générale, on peut constater l'extrême difficulté qu'ont ces jeunes adultes à mettre fin à toute relation avec leurs parents. Ainsi, Katia, en procès contre ses parents en raison de leurs violences passées, continue pourtant à voir sa mère. Cette difficulté peut être reliée à la force anthropologique du lien qui, malgré le caractère douloureux des relations, continue à unir - à emprisonner ? - parents et enfants. L'histoire de Samuel nous en donne un exemple éclairant. II a quitté ses parents dans un climat très difficile: "Mon départ de chez moi, ça a été fort, ça a été dur. C'était un désaccord complet avec ma mère et mon beau-père. Sur la manière de vivre, de m'éduquer, de ne pas me laisser grandir... Ce sont des rapports anciens avec ma mère, il y a des choses que je n'ai jamais pu lui pardonner, je n'y arrive pas. Elle n'a pas fait son mea culpa, elle n'est pas capable de le faire, je pense qu'elle n'en sera jamais capable, comme sa mère ne l'a jamais fait (...). J'ai eu besoin de partir ». Samuel est parti vivre avec sa grand-mère, mais là aussi le conflit couve... "J'en avais un peu parlé avec ma grand-mère. Le but n'était pas de m'accueillir parce que je suis son petit-fils et pour m'aider, le but, c'est de faire du mal à sa fille. Donc c'est perfide. J'ai fait le mauvais choix en partant chez ma grand-mère, mais je n'avais pas d'autres possibilités matérielles et financières. Ma mère n'a pas du tout apprécié (...). Ce sont des gens avec qui je ne peux pas m'entendre. À la limite, ils voulaient que la coupure soit nette mais, moi, j'avais encore besoin d'eux. Je pense que, bientôt, et ça ne traînera sans doute pas, je ne les verrai carrément plus...».

En troisième vague d'enquête, Samuel a quitté sa grand-mère, a vécu seul et vient de s'installer avec sa compagne. Il a cependant encore du mal à vraiment couper les liens qui le font souffrir : "Et puis ma mère, je la vois ponctuellement aussi. Si je ne le 
l'appelle pas, elle ne m'appellera pas. Je n'attends rien en fait... C'est uniquement pour être dans la norme ou parce qu'il me faut encore cette norme pour exister. Après, quand je saurai m'en passer, quand j'aurai la force, je pourrai me le permettre (...). Mais ça ne me fait pas peur, et puis il le faudra parce que ce lien n'est vraiment pas fort et nous fait peut-être plus souffrir qu'il ne nous apporte des choses, des deux côtés ». La " norme» est tout ce qui le tient aujourd'hui dans cette relation, la norme mais aussi, de moins en moins cependant, le manque et la souffrance. La décohabitation certes soulage, mais ne règle pas la question de ces liens si cruciaux pour la construction identitaire (Déchaux, 1997 ; Favart, 2005). En quatrième vague, Samuel vit à nouveau seul. Du côté de sa mère la rupture est consommée : «Les autres moments forts, ça a été la rupture avec ma mère et mon beau-père il y a huit ou neuf mois. J'ai coupé. (...) C'est difficile, c'est toujours difficile à vivre parce que je me rends compte que j'ai du mal à construire. Pourtant, j'ai toujours grandi sans eux donc sans avoir à écouter leurs conseils, parce qu'ils n'en avaient pas... mais on a quand même besoin, je pense, d'avoir ses parents, sentir qu'on est épaulé. Là, je ne suis pas épaulé, pas du tout ».

Même quand la rupture de la relation a été enfin accomplie, le lien se fait toujours sentir, par défaut, par son absence qui reste douloureuse. On voit bien là tout le poids de ce lien, un poids qui s'avère pour partie indépendant de la qualité de la relation: Si celle-ci est douloureuse, le lien demeure néanmoins difficile à rompre, et s'il est rompu, il se manifeste toujours telle une charge pesant encore « en creux ». Certes, le processus de cheminement vers l'âge adulte est relationnel, certes, il implique une redisposition des places entre les générations. Mais tout ne s'y négocie pas, dans la mesure où le lien de famille tient sa place quelles que soient la réalité vécue et la qualité affective de la relation.

\section{Devenir adulte : une mise à l'épreuve des rapports intergénérationnels}

Lorsqu'on pose à ces jeunes la question "Dirais-tu que tu es adulte? Pourquoi?", la réponse mais surtout ses motivations sont intéressantes à plus d'un titre, son évolution dans le temps également (Bidart, 2005). Le rapport intergénérationnel est de fait amplement mobilisé dans l'argumentaire. Devenir adulte, c'est avant tout un processus qui engage les rapports avec ses parents, qui engage aussi le projet de devenir soi-même un parent. S'il n'est pas certain que le sens du mot « adulte » soit bien solide, son évocation et son argumentation (ce qui nous intéresse le plus finalement) mobilisent surtout la référence au cycle des générations. Devenir adulte, c'est le devenir avec, contre ou aux yeux de ses parents. Cette mutation engage certes le lien dont on a parlé plus haut, mais implique également une transformation de la relation avec les parents.

\section{Adulte comme les parents, adulte aux yeux des parents}

Lors de cette période charnière de la jeunesse qui marque la transition entre deux générations, celle des parents du jeune et celle des enfants qu'il ou elle se prépare éventuellement à mettre au monde, le lien mais aussi la relation entre parents et enfants vont "bouger». Si les parents sont fortement mobilisés sur le plan symbolique, ils vont aussi être mis à l'épreuve par certaines formes de renversements. Certes, le rapport intergénérationnel se fonde avant tout sur un lien qui résiste et s'impose à l'histoire. Dans ce sens, tout dans ces transformations ne relève pas de la négociation. L'écart irréductible entre les générations fait partie des éléments qui résistent. Kévin l'exprime à sa façon: "Je suis adulte au niveau de la loi et au niveau de ce que je fais comme activité, comme boulot, ça fait des responsabilités. Je suis adulte au niveau de ça, que je paie mon loyer, que je m'assume, mais je ne pense pas être adulte. Moi, j'ai besoin de gens plus vieux que moi. Donc, tant que j'ai besoin de gens plus vieux que moi, parce qu'on a toujours besoin d'eux, ils sont toujours plus adultes que moi. Je vois ça comme ça (...). À la rigueur, un adulte je dirais que c'est mon père. Et vu que mon père sera toujours là, il sera toujours plus vieux que moi...".

Parfois, les parents contribuent à cet ordre incontournable, ainsi que l'exprime Émeline: "Je ne peux pas me considérer comme adulte parce que mes parents ne me le font pas sentir comme tel. Mais, en même temps, je ne suis pas sûre que ça change un jour. Ma mère me considérera toujours comme son idiote de fille immature ».

Cependant, au fil du temps, certaines choses peuvent bouger. II s'agit moins de combler cet écart que de le dépasser et de se situer soi même dans une place d'adulte, une place toute proche de celle des parents, comme pour Gaël: "Avant, quand on disait "Monsieur P.", je disais : "Mon père n'est pas là". Je pensais à mon père, Monsieur $P$. Maintenant, quand on dit: "Monsieur P.", c'est moi ! ». Le rôle des parents dans cette appréciation est là encore primordial. On devient adulte avant tout à leurs yeux, comme le confirme Alban : "C'est les avantages, les gros avantages d'être adulte, le regard qu'on porte sur vous. Dans la famille, par exemple, c'est quand même beaucoup plus de respect».

Ce respect relève plus de la relation que du lien. II implique que l'évolution autorise une certaine distance rendant possible l'émergence d'une relation entre égaux. C'est ce qu'exprime Katia qui tente de 
sortir des rôles mère-fille: "Avec ma mère, je suis devenue plus adulte il n'y a pas si longtemps que ça. J'ai dit à ma mère que ça suffisait, que je vivais chez moi, que j'avais ma vie, que j'en avais marre, qu'il fallait qu'elle essaie de me voir différemment parce qu'elle essayait de me donner tout le temps de l'argent pour m'aider parce qu'on avait quelquefois des problèmes d'argent (...). Même si à une époque je m'entendais mal avec elle, j'avais tendance à l'appeler quand ça n'allait pas. Mais maintenant, de moins en moins, parce que je voudrais qu'elle me lâche un peu. C'est peut-être dur, je la comprends aussi : ce qu'elle fait, ce qu'elle dit, c'est normal, c'est une mère avant tout, on ne pourra rien y faire. Mais, moi, j'avais besoin qu'elle me laisse faire ce dont j'avais envie ». Le message qu'envoient bien des jeunes : "C'est une mère, on ne peut rien y faire, mais cette mère-là, aujourd'hui, doit parvenir à m'accepter comme adulte ", témoigne ainsi de cette différenciation entre lien et relation.

\section{Adulte dans la relation avec les parents}

Dans ce processus, il semblerait qu'au lien inamovible puisse peu à peu s'ajouter une dimension relationnelle de plus en plus étoffée, de plus en plus élaborée sur des interactions et des évaluations interpersonnelles. La relation ne se substitue pas pour autant au lien, elle s'y superpose plutôt, elle l'enrobe, I'accompagne. C'est dans la relation avec leurs parents que les enfants peuvent alors " négocier» la reconnaissance et s'inscrire dans des places comparables en dignité à celle de leurs parents. On peut percevoir cette évolution sur deux plans. Tout d'abord, l'accès à l'âge adulte s'accompagne d'une transformation du régime de la parole: devenir adulte, c'est pouvoir parler avec ses parents, leur dire des choses, être entendu par eux. Paul explique l'évolution de sa relation avec son père: "Je ne répondais pas trop, je ne m'imposais pas trop (...). Maintenant, je parle beaucoup plus, aussi bien sur les conneries que je peux faire, parce qu'on a fini d'être gamin et de les cacher. C'est pareil, je suis franc avec lui, si il y a un truc qui ne me plaît pas, je sais que ça va le mettre en rogne que je lui dise, mais je le dis et je sais qu'ils apprécient de me voir mûrir (...). Je sais qu'au fond il appréciera, même si ça l'énerve».

Lorsque cette nouvelle modalité d'échange n'intervient pas, le processus reste bloqué, comme pour Viviane qui pourtant travaille, a acheté un appartement et attend un enfant : "Par exemple, quand on me parle de mes parents, je n'arrive pas à être adulte, parce que je n'arrive pas à dire à mes parents ce que je pense d'eux. Quand on parle avec mes parents, je n'arrive pas à être adulte (...). Mes parents ne me connaissent pas en tant $q u$ 'adulte en fin de compte (...). Je n'arrive pas à discuter réellement avec mes parents, à leur dire ce que je pense d'eux vraiment, que je ne les supporte plus. Je les aime, mais je ne les supporte plus, je ne pourrais pas les supporter, j'ai beaucoup de choses qui... pfff !».

Entrer dans l'âge adulte, c'est aussi et dans le même mouvement finir par voir ses parents comme des personnes, les sortir de leur rôle et, pour partie du moins, leur donner une dimension humaine. Luc exprime bien cette mutation : « $A u$ début, je pensais que, vu que ma mère faisait beaucoup de choses, je pensais que ma mère n'était pas faillible. Et quand elle a eu ses problèmes de santé, j'ai compris qu'elle n'était pas supérieure aux autres. Au début, je pense que j'identifiais un peu ma mère comme une déesse. Et avec ses problèmes de santé, elle est revenue au rang des mortels; donc j'ai vu qu'elle était comme tout le monde. Ça m'a fait réfléchir sur pas mal de choses (...). Je la mettais sur un piédestal, je n'osais pas du tout discuter avec elle, tout ce qu'elle disait était vrai. Petit à petit, j'ai compris qu'on avait le droit aussi d'exister. Et elle a commencé à évoquer certains sujets sur sa vie avant, avant ma venue. On n'était pas du tout au courant de ça, et ça nous a éclairés sur plein de choses, sur certaines réactions. On a compris».

Il ne s'agit pas d'un changement de nature du rapport entre parents et enfants, mais d'une complexification et d'une évolution multidimensionnelle : on " ajoute» de la relation au lien. Mais celui-ci n'est pas loin, reste présent et actif, comme on le voit dans les situations difficiles évoquées plus haut, mais aussi dans certaines ambivalences typiques $d u$ «saut » intergénérationnel. On voit ainsi bien des jeunes adultes responsables redevenir des enfants auprès de leurs parents, retrouver le lien " anthropologique » dans certaines circonstances. $C^{\prime}$ est le cas d'Étienne, père de famille, qui peut jouer sur les deux tableaux : "Je suis plus responsable, maintenant avec les enfants, on n'a pas le choix de toute façon. Et puis plus réfléchi. On réfléchit plus à ce qu'on va faire, on ne part pas tête baissée en se disant qu'on fonce. Et en quoi je suis encore enfant, c'est quand je vais chez mes parents, il y a le petit cocon familial qui est là et puis je repasse au statut d'enfant, malgré qu'on aie des réflexions d'adulte, on peut discuter de choses et d'autres entre adultes, mais le petit cocon fait que je reste... ». La transition vers la vie adulte montre clairement sa dimension relationnelle, relative et évolutive, pour partie fondée sur les interactions et les confrontations avec les parents.

\section{Adulte comme qui ? Inversion des rôles et difficultés du passage}

En conséquence, les difficultés éprouvées dans les relations mais aussi dans les liens intergénérationnels ne sont pas sans effet sur cette transition. 
Lorsque la vie est dure, lorsque les rapports parent-enfant sont perturbés, le positionnement par rapport à l'âge adulte, qui est on l'a vu fortement référencé par les parents, s'en ressent. II est en particulier difficile de s'assimiler à un adulte lorsque la génération des parents, modèle par excellence, a "failli ». Le cas de Diane est éclairant à cet égard. Son histoire a été évoquée plus haut à propos de sa décohabitation bloquée par la prise en charge de sa mère alcoolique et dépressive, une mère qui n'assume pas son rôle d'adulte. Diane, trois ans après, a du mal à parler de ce qu'est un adulte: (Est-ce que tu considères aujourd'hui que tu es adulte?) "Je ne sais pas, parce que je ne vois pas ce que c'est adulte, déjà. Je ne vois pas trop ce que ça veut dire (...). Je n'ai pas envie de dire que je suis adulte alors que je n'ai pas envie d'être adulte (C'est quoi l'image qui ne te plaît pas ?) : C'est l'image de mes parents (Tu n'as pas envie d'être comme ça ?): Non. Parce que ma mère était alcoolique, parce que mon père est parti trop tôt, parce qu'ils ne m'ont pas montré des choses que j'espère montrer à mes enfants...».

Encore trois ans après, Diane est enceinte mais parle peu de sa grossesse (ce qui est rare) et déclare à propos de l'entretien qui se termine : "Ça m'a permis de faire un bilan et de me dire que, maintenant, je vais être maman. Voilà, je vais être maman. Parce que je n'arrivais pas trop à me le dire. Et là, c'est ce qui ressort vraiment. C'est la première fois que je dis que je vais être maman».

Ainsi, le fait de voir les rôles inversés, d'avoir à assumer les problèmes des parents, de devoir les remplacer parfois, implique que l'on doute d'eux parfois un peu trop tôt, et qu'il devient alors difficile de savoir clairement ce qu'est un adulte. Le divorce, le veuvage rendent également parfois les parents très fragiles, surtout lorsque cela s'ajoute à une vulnérabilité sociale. Ces épreuves vécues par le biais de leurs parents recèlent pour les jeunes un caractère initiatique, mais sans toujours révéler un modèle bien clair derrière les remises en cause qu'elles imposent.

\section{Devenir soi-même parent}

Au-delà de I'histoire de Diane, la parentalité recouvre le point ultime de la transition vers l'âge adulte. Que les jeunes en parlent en creux ou en plein, que cet événement soit repoussé, envisagé ou assumé, le fait d'avoir un enfant est crucial dans la transformation du rapport intergénérationnel. Sans qu'il soit possible de développer et d'illustrer l'ensemble de ces enjeux, on souhaite insister ici sur un point bien précis. Dans tous les entretiens apparaît la liaison très forte entre les rapports avec les parents et le projet de faire un enfant. En effet, les deux dimensions sont étroitement associées. Faire un enfant, c'est devenir soi-même parent, et c'est « régler » cela au regard de ses propres parents. C'est pourquoi il est important de prendre en considération la double dimension intergénérationnelle, en associant dans les travaux en sciences humaines ces deux moments du rapport de filiation, ces deux liens fondamentaux, ces deux modalités imbriquées : avoir des parents et avoir des enfants.

Solange montre bien cette dialectique : "Je pense que je serai adulte quand j'aurai réellement passé le flambeau et créé une famille. Je pense que là tu entres dans le monde des adultes. Pour moi, je pense qu'il manque un seuil. Parce que j'ai une vie professionnelle, j'ai une vie privée, je me sens bien et je pense avoir les mêmes moments, les mêmes passages que mes parents et vivre comme un adulte. Ils rentrent tous les soirs du travail et ils parlent du travail, ils vivent grâce à ce qu'ils font en journée, ils n'ont de compte à rendre à personne. Je sais prendre des décisions, je sais faire des choix, j'assume ce que je fais. II reste juste à savoir si je suis capable d'être parent, tout simplement ". Violette, enceinte, rend compte elle aussi du double mouvement qu'implique la venue de l'enfant: " C'est nous qui devrons prendre les bonnes décisions pour l'enfant, comme nos parents ont fait pour nous. On avance d'un cran aussi. On est des enfants, mais on va être parents. Parce qu'on est toujours des enfants pour nos parents, mais on est autre chose en plus. C'est un deuxième statut, parent et enfant».

Là encore, les problèmes de famille montrent toute leur pesanteur et leur impact, lorsqu'ils viennent directement perturber le projet de filiation. Samuel, dont nous avons mentionné plus haut la difficulté à rompre avec sa mère, en tire les conséquences et refuse d'avoir un enfant: "Je ne veux pas leur donner ce que je n'ai pas eu : je ne veux pas donner à ma mère des petits-enfants qu'elle va adorer et pour qui elle serait une bonne grand-mère. C'est dur, mais j'en souffrirais, et ça pourrait se reporter sur les enfants ». La reproduction lui fait peur, dans tous les sens du terme... Les deux rapports sont bien liés pour lui, celui avec la génération des parents et celui avec la génération des enfants. Rose tire la même conclusion : "Déjà je ne veux pas de gosses donc, c'est bon... Oui, non, je crois que j'ai trop souffert avec ma famille, je ne voudrais pas qu'un gosse souffre autant que j'ai fait. Non, je ne pense pas. Je me dis, il aura toujours des problèmes avec la mienne, toujours, quoi qu'il se passe, parce que même moi, même en partant, j'en ai toujours donc je ne veux pas que le gosse il souffre autant que ce que j'ai vécu et automatiquement, il l'aura 
puisque ma famille est comme ça et ne changera jamais. Donc je me dis, non, il vaut mieux pas. Je m'occuperai de ceux de ma cousine s'il faut, mais, non, je ne veux pas de gosse».

\section{La relation à trois générations}

La plupart du temps, le fait d'avoir un enfant transforme positivement le rapport intergénérationnel. Parce qu'il établit une équivalence entre la place des parents et celle des enfants (en devenant parents, les jeunes sont devenus " comme » leurs parents), il permet, facilite ou renforce leur relation. Ainsi Julien constate-t-il que : «La priorité de mes parents, c'est les enfants, et ma priorité, c'est mes enfants et ma famille. C'est sûr qu'on se rejoint parce que, mine de rien, il y a vraiment un noyau ». Amélie observe, elle aussi, les effets bénéfiques de cette convergence: "Depuis que j'ai Lou, ça va beaucoup mieux avec lui [mon père], parce qu'il est en train de s'apercevoir que je m'aperçois aussi de ce que c'est qu'avoir un enfant ». Lorsque les relations avec les parents sont douloureuses, l'arrivée d'un enfant, si elle n'améliore pas nécessairement les rapports intergénérationnels, empêche cependant leur rupture. En effet, l'existence d'une relation entre les petits-enfants et leurs grands-parents est généralement présentée comme un «droit » que les nouveaux parents se doivent de respecter, et ce quelle que soit leur propre relation avec leurs géniteurs. C'est ce qu'explique Jérémie: "Elle est maligne, ma mère. Elle joue beaucoup sur Caroline [la fille de Jérémie]. (...) Même si on s'embrouille, il y a toujours la gamine pour nous ramener (...). On ne peut pas dire à la gamine "tu ne verras plus tes grands-parents !" ».

Au terme de ce parcours, il semble donc que si le cheminement vers l'âge adulte consiste, pour les parents comme pour les enfants, à essayer $d^{\prime}$ « ajouter» de la relation au lien qui les unit, le fait, pour les enfants, de devenir parents à leur tour améliore le rapport intergénérationnel en ce qu'il favorise la relation ou empêche de la rompre en raison de cet autre lien unissant petits-enfants et grands-parents. Lien " non négociable » car ancré dans la conception moderne (Singly, 2003) selon laquelle la singularité de l'individu prend sa source dans son histoire, dans son origine et qu'elle se nourrit donc, entre autres, des relations avec les grands-parents.

\section{La double dimension intergénérationnelle}

L'époque contemporaine voit se confirmer une complexification du cheminement vers l'âge adulte dans un contexte de transformation des rapports entre générations : devenir adulte, ce n'est pas se libérer brusquement de la tutelle des parents en quittant définitivement leur foyer mais c'est plutôt expérimenter avec eux un long processus relationnel qui peu à peu contribue à construire une certaine autonomie. En conséquence le «devenir adulte» implique, de plus en plus peut-être, les rapports intergénérationnels. On a pu à cet égard parler d'une relative "libéralisation» des rapports de famille : la modernité transformerait les liens et les obligations de famille en relations électives et affectives avant tout. L'enquête a permis de nuancer cette analyse. En effet, si la parenté autorise sans doute plus qu'autrefois la mise en œuvre de relations diversement épanouies, elle reste néanmoins un lien structurel, anthropologique, résistant aux aléas des interactions entre ses membres. La distinction entre lien et relation à propos de la famille semble donc tout à fait nécessaire.

La force du lien ressort tout particulièrement lorsqu'il est mis à l'épreuve, à savoir dans les cas de rapports douloureux mais aussi au moment où les jeunes adultes deviennent eux-mêmes des parents, lorsqu'intervient la " bascule » générationnelle. Les jeunes occupant alors la même place que leurs parents auparavant, les rôles et les regards mutuels se modifiant, une relation plus égalitaire peut émerger ; la parole se dénoue, les personnes s'affirment. Par ailleurs, le projet de faire des enfants et les rapports avec les parents restent, pour ces jeunes, très étroitement liés entre eux, ce qui invite à prendre en considération la «double dimension intergénérationnelle» de cette transition. Devenir adulte, $c^{\prime}$ est donc peut-être avant tout s'inscrire dans cette double dimension intergénérationnelle par la reconnaissance des liens et construire, "en plus", des relations interpersonnelles avec ces autruis pas comme les autres que sont les parents. 


\section{Références bibliographiques}

Battagliola F., 1997, Itinéraires de passage à l'âge adulte; différences de sexe, différences de classe, Sociétés contemporaines, $n^{\circ}$ 25:85-103.

Bidart C. (dir.), 2006, Devenir adulte aujourd'hui : perspectives internationales, INJEP, L'Harmattan, collection Débats jeunesse.

Bidart C., 2005, Temps de la vie et cheminement vers l'âge adulte, Lien social et politiques, n 54:51-63.

Bidart C., 1997, L'amitié, un lien social, La découverte.

Bidart C. et Lavenu D., 2006, Transitions vers la vie adulte et origines sociales. Une enquête longitudinale en France, in Devenir adulte aujourd'hui : perspectives internationales (sous la dir. de Bidart C.), INJEP, L'Harmattan, collection Débats Jeunesse:163-180.

Blöss T., 1997, Les liens de famille. Sociologie des rapports entre générations, PUF.

Chamboredon J.-C., 1985, Adolescence et post-adolescence : la "juvénisation », in Adolescence terminée, adolescence interminable (sous la dir. de Alléon M., Morvan O. et Lebovici S.), PUF.

Chauvel L., 1998, Le destin des générations, PUF, collection Le lien social.

Cicchelli V., 2001 a, La dépendance familiale des jeunes adultes en France et en Italie, Recherches et Prévisions, $n^{\circ}$ 65:31-37.

Cicchelli V., 2001 b, Les jeunes adultes comme objet théorique, Recherches et Prévisions, n 65:5-18.

Cicchelli V., 2001 c, La construction de l'autonomie. Parents et jeunes adultes face aux études, PUF.

Déchaux J.-H., 1997, Le souvenir des morts. Essai sur le lien de filiation, PUF.

Dubar C., 1995, La socialisation. Construction des identités sociales et professionnelles, Armand Colin, collection U.

Favart E., 2005, Parcours de vie et mémoires familiales, in Parcours de vie ; regards croisés sur la construction des biographies contemporaines (sous la dir. de Guillaume J.-F., Lalive d'Epinay C. et Thomsin L.), Éditions de I'Université de Liège.

Galland O., 2001, Adolescence, post-adolescence, jeunesse : retour sur quelques interprétations, Revue française de sociologie, volume XLII, $n^{\circ}$ 4:611-640.

Galland O., 1990, Un nouvel âge de la vie, Revue française de sociologie, volume XXXI, n 4:529-551.

Le Gall D., 1998, Family conflicts in France through the eyes of teenagers, in Multidisciplinary perspectives on family violence in Europe, sous la direction de Renate Klein, Routledge, London, England.

Le Gall D. et Pellissier A., 2001, Que manque-t-il à l'amour pour faire couple?, Agora/Débats Jeunesse, $n^{\circ}$ 23:9-23. 
Mauger G., 1994, Jeunesses et société, Armand Colin.

Pellissier A., 2002, La première fois sera-t-elle aussi la dernière ? Trajectoires de décohabitation et cheminements vers l'âge adulte, Agora/Débats Jeunesse, n² 28:80-92.

Ramos E., 2002, Rester enfant, devenir adulte: la cohabitation des étudiants chez leurs parents, L'Harmattan, collection Logiques sociales.

Rose J., 1998, Les jeunes face à l'emploi, Desclée de Brouwer.

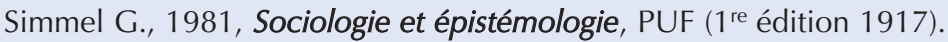

Singly F. (de), 2003, Les uns avec les autres. Quand l'individualisme crée du lien, Armand Colin.

Singly F. (de), 2001, Etre soi parmi les autres - Famille et individualisation, L'Harmattan.

Singly F. (de), 2000, Penser autrement la jeunesse, Lien social et politiques, $\mathrm{n}^{\circ}$ 43:9-21.

Théry I., 1998, Couple, filiation et parenté aujourd'hui. Le droit face aux mutations de la famille et de la vie privée, Odile Jacob.

Van de Velde C, 2004, "Devenir adulte : sociologie comparée de la jeunesse en Europe », thèse pour le doctorat de sociologie, Institut d'études politiques Paris 3. 
